\title{
Detection of IEMI Fault Injection Using Voltage Monitor Constructed with Fully Digital Circuit
}

\author{
Daisuke Fujimoto, Yu-ichi Hayashi \\ Nara Institute of Science and Technology, Japan \\ fujimoto@is.naist.jp,yu-ichi@is.naist.jp
}

\begin{abstract}
A threat to intentionally induce electromagnetic waves inside the cryptographic integrated circuit (IC) exists, which may cause temporary faults in processing inside the IC to acquire a secret key. We propose a method of suppressing leakage of the secret key to protect equipment from such attacks by monitoring the interfering wave inside the IC. In the proposed method, voltage variations in the amplitude and time inside the IC are measured, and an attack is detected. Further, to cheaply and easily implement the proposed method, the monitor consists of a fully digital circuit.
\end{abstract}

Keywords-EM information leakage; fault injection attacks; intentional electromagnetic interference; digital voltage monitor;

\section{INTRODUCTION}

In recent year, Internet-of-things (IoT) devices have been widely used in various situations. IoT devices use a network for communication. When the network is used, guaranteeing confidentiality and integrity of the data is an important task, and the security of such data is ensured using cryptographic devices. Therefore, the role of cryptographic devices becomes very important.

On the other hand, problems of security degradation due to physical attacks on cryptographic devices have been pointed out in recent years. In particular, fault injection attack using intentional electromagnetic interference (IEMI) is one of the problems where countermeasure is urgently needed. These attacks induce interfering waves inside the integrated circuit (IC) through the power and communication lines connected to the equipment and cause faults (Fig. 1) [1,2]. These threats may extend to IoT devices that have been widely used in recent years. Because these devices operate with reduced power compared with conventional equipment, their immunity is believed to have decreased.

We propose a detection method of IEMI fault injection using on-chip voltmeter against this type of threats, which consists of a low-cost digital circuit, because a possibility exists that leakage of secret information can be suppressed if interference waves that could cause faults in the cryptographic module can be detected. Because conduction disturbance waves are used in the attacks, we can detect frequencies of less than $30 \mathrm{MHz}$, which are defined as conduction disturbance in Federal Communications Commission (FCC) Part 15 [3] and International Special Committee on Radio Interference (CISPR) 22 [4].

\author{
Arthur Beckers, Josep Balasch, Benedikt Gierlichs, \\ Ingrid Verbauwhede \\ ESAT-COSIC, KU Leuven, Belgium
}

The structure of this paper is described as follows. Section II proposes a chip voltage-measurement method using digital circuits and a fault-estimation method based on IEMI. Section III presents the actual evaluation of the implementation on a field-programmable gate array (FPGA), and the summary is provided in Section IV.

\section{DETECTION METHOD OF IEMI FAULT}

In this section, we describe the mechanism of occurrence of faults that may cause leakage of secret keys. To detect faults, we clarify the physical quantity to be monitored inside the IC. We also propose methods to monitor these faults.

\section{A. Fault mechanism in a cryptographic core}

To detect a fault, we need to investigate what type of signal causes the fault in a cryptographic core. The causes of faults are roughly divided into lower power supply and overclocking [5,6] (Fig. 2). The circuit operation is delayed because of the reduction in the power supply. The processing is not completed because of the speed reduction following a low power supply, and fault occurs.

Overclocking causes a fault by superimposing an EM pulse on a signal and causing the circuit to exceed the threshold before the normal clock cycle. Countermeasures against overclocking include clock-time and pulse measurements $[7,8]$. As described earlier, to deal with faults due to multiple factors, existing countermeasures require multiple countermeasure circuits. Our countermeasure circuit aims at detecting multiple types of fault using the same circuit from the viewpoint of power-supply voltage measurement.

\section{B. Fault detection using voltage measurement in a chip}

A fault in an IC occurs because of the drop in the powersupply voltage or superimposition of noise on the clock signal line. Attackers attack using the power line; thus, the superimposition of noise on the signal propagates from the noise in the power line (Fig. 3). Therefore, a mechanism for estimating the power-supply voltage within the chip is required. Many power-supply voltage-measurement circuits using analog circuits have been proposed. However, for them to be installed in IoT equipment, we need to reduce the circuit cost. Therefore, we employ a circuit for measuring the powersupply voltage using a digital circuit. 
We know that the delay of the digital circuit depends on the power-supply voltage, as shown in equation (1) $[9,10,11]$.

$$
\text { delay } \propto \frac{L^{2} V_{d d}}{\mu\left(V_{d d}-V_{t h}\right)^{\alpha}}
$$

The power-supply voltage measurement by the digital circuit with this voltage dependence can be measured using a delay chain circuit in which the delay elements are connected in series, as shown in Fig. 3. When the same clock is delayed and sampled, we can possibly obtain an output according to the delay amount. Because the clock signal periodically changes, it is suitable for estimating the delay amount.

To detect the drop in the power-supply voltage, we measure the average voltage per clock cycle and check whether it is lower than the power-supply voltage when the fault occurs. We only need to periodically check the output of the above-described circuit. With respect to overclocking, attacks through the power lines from a distance can be foreseen by checking whether intentional signals with a certain amplitude are superimposed on the power-supply line. Once the security device determines an intentional signal, countermeasure can be considered at the system level.

\section{EXPERIMENT}

\section{A. Experimental setup}

We develop the proposed digital voltage meter on Degilent's ARTY FPGA board. The delay element consists of two NOT circuits composed of lookup tables, which is a primitive cell in the FPGA. The delay line is composed of 32 delay elements. Each $100-\mathrm{MHz}$ sampling clock value is stored in the register. Every 100 records are transferred to a PC for evaluation. The supply voltage is supplied from a stable power supply (PA18-3B), and a sinusoidal waveform, which is directly injected to the power line, is generated by a signal generator (Fig. 4).

\section{B. Detection of fault caused by static voltage drop}

Table 1 lists the output value of the voltage monitor by sweeping the power-supply voltage. This result suggests that the prototype design has voltage resolution of approximately $10 \mathrm{mV}$. Because the normal power-supply voltage is $0.95 \mathrm{~V}$, a part exists where the voltage resolution decreases at a voltage higher than 0.95 V. The Advanced Encryption Standard (AES) core implemented in ARTY outputs a faulty output at an external supply voltage of $0.80 \mathrm{~V}$. To prevent fault operation, the voltmeter issues an alert at a certain margin from $0.80 \mathrm{~V}$ to the system.

\section{Detection of fault caused by IEMI signal injection}

To verify that signal injection from outside can be detected, a $5-\mathrm{V}_{\mathrm{pp}}$ signal of $10 \mathrm{MHz}$ is injected from the signal generator. The output of the voltage monitor at this instant is listed in Table 2. A peak appears every 10 points of the $100-\mathrm{MHz}$ sampling clock. Detecting the injected signal is possible from the peak to peak values after confirming the voltage change in such continuous time. The equivalent circuit of the experiment is shown in Fig. 5. The injected signal on board with $90 \mathrm{mV}$ is

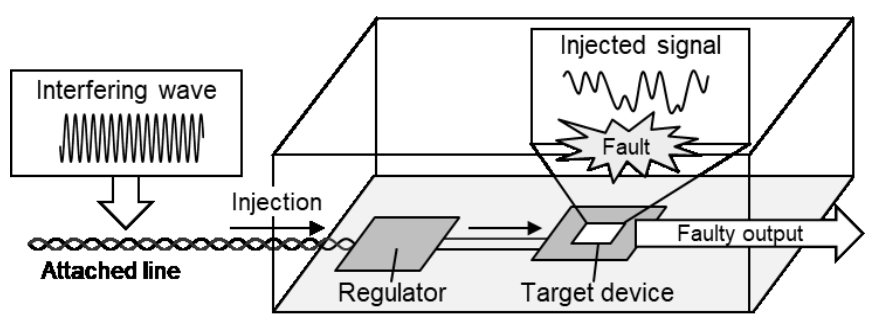

Fig. 1 Image of IEMI fault injection

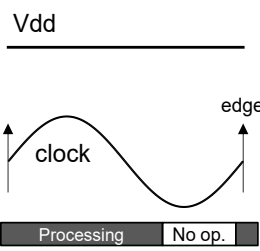

(a) Normal condition

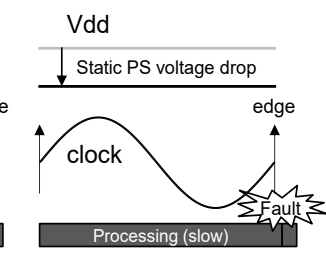

(b) Low power supply
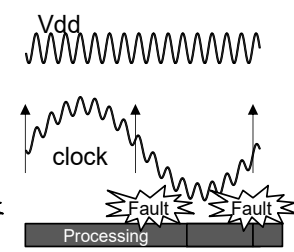

(c) Signal Injection

Fig. 2 Image of fault on crypto circuit with IEMI injection

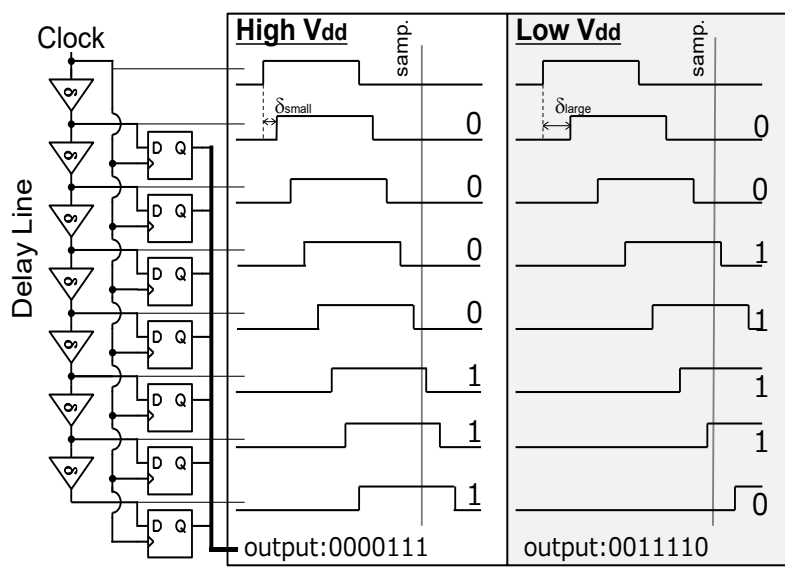

Fig. 3 Schematic of the digital voltage monitor and the output image

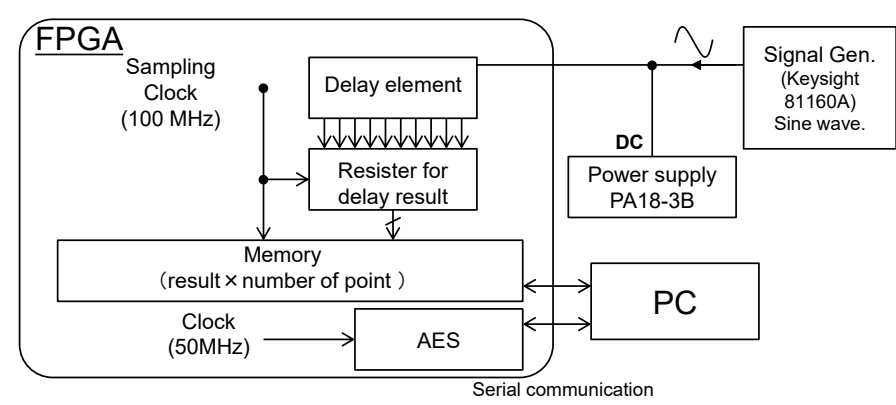

Fig. 4 Prototype implementation and block diagram of the experimental setup

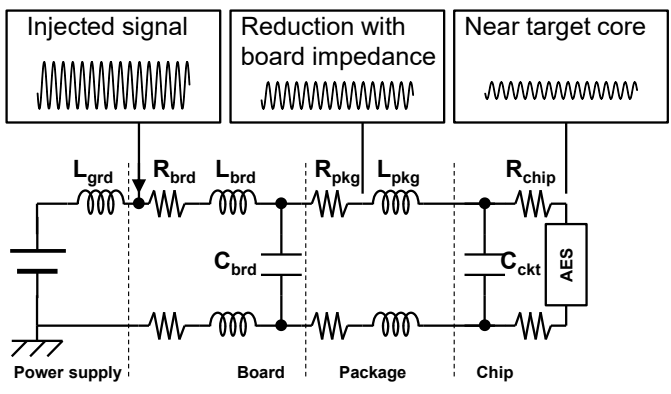

Fig. 5 Image of the IEMI fault injection 
TABLE 1 VOLTAGE CODE OUTPUT DEPENDING ON THE POWER SUPPLY

\begin{tabular}{|l|l|}
\hline Power supply (V) & Voltmeter code \\
\hline 0.85 & 00011111111000000011111111000000 \\
\hline 0.86 & 01111111100000000011111111000000 \\
\hline 0.87 & 11111111100000000111111111000000 \\
\hline 0.88 & 11111111000000000111111100000000 \\
\hline 0.89 & 11111110000000011111111100000000 \\
\hline 0.90 & 11111100000000011111111100000000 \\
\hline 0.91 & 11111000000000011111111100000000 \\
\hline 0.92 & 11110000000000011111111100000000 \\
\hline 0.93 & 11100000000000111111111000000000 \\
\hline 0.94 & 11000000000001111111111000000000 \\
\hline 0.95 & 10000000000001111111111000000000 \\
\hline 0.96 & 00000000000011111111111000000000 \\
\hline 0.97 & 00000000000111111111110000000000 \\
\hline 0.98 & 0000000000011111111110000000000 \\
\hline 0.99 & 0000000000111111111110000000000 \\
\hline 1.00 & 0000000001111111111110000000000 \\
\hline & \\
\hline
\end{tabular}

TABLE 2 VOLTAGE CODE OUTPUT WITH IEMI INJECTION

\begin{tabular}{|l|l|l|l|}
\hline Points & Voltmeter code (half) & Points & Voltmeter code (half) \\
\hline 1 & 0000001111111000 & 11 & 0000001111111000 \\
\hline 2 & 0000001111111000 & 12 & 0000001111111000 \\
\hline 3 & 0000011111110000 & 13 & 0000001111111000 \\
\hline 4 & 0000111111100000 & 14 & 0000011111110000 \\
\hline 5 & 0001111111100000 & 15 & 0001111111100000 \\
\hline 6 & 0001111111100000 & 16 & 0001111111100000 \\
\hline 7 & 0000111111100000 & 17 & 0000011111110000 \\
\hline 8 & 0000011111111000 & 18 & 000001111110000 \\
\hline 9 & 0000001111111000 & 19 & 0000001111111000 \\
\hline 10 & 0000001111111000 & 20 & 0000001111111000 \\
\hline
\end{tabular}

observed. In the chip, the injected signal is reduced to $30 \mathrm{mV}$ with the chip impedance. In this result, the voltage monitor can capture small injected signals. Therefore, the voltage monitors could possibly capture larger signals that cause faults in the cryptographic core.

\section{CONCLUSION}

In this paper, we have proposed a voltage monitor to detect IEMI fault injection threats to cryptographic cores. The developed monitor was constructed using a fully digital circuit, and if a programmable area is available in the system, this function can be installed in an IoT device even after product shipment. In addition, because the developed circuit can monitor the voltage inside an IC in the range from 0.85 to 1.00 $\mathrm{V}$, cryptographic devices can possibly detect the interference before issuing faulty outputs used for fault analysis. In the future, we plan to implement the developed monitor on an actual IoT device and examine the possibility of actual attack detection.

\section{REFERENCES}

[1] Y. Hayashi, N. Homma, T. Mizuki, T. Aoki, and H. Sone, "Transient IEMI Threats for Cryptographic Devices," IEEE Trans. on Electromagnetic Compatibility, Vol. 55, No. 1, pp. 140-148, 2013.

[2] K. Iokibe, K. Maeshima, H, Kagotani, Y. Nogami, Y. Toyota, and T. Watanabe, (2014, August). Investigation in burst pulse injection method for fault based cryptanalysis. IEEE International Symposium on Electromagnetic Compatibility (EMC), (pp. 743-747) 2014.

[3] Federal Communications Commission, "FCC Part 15 Subpart B".

[4] CISPR, "CISPR 22 Radiated and Conducted EMI Limits".

[5] G. Piret, and J.J. Quisquater, "A differential fault attack technique against SPN structure, with Application to the AES KHAZAD," Cryptographic Hardware and Embedded Systems (CHES), pp.77-88, 2003.

[6] S. Guilley, L. Sauvage, J.L. Danger, N. Selmane, and R. Pacalet, "Silicon-level solutions to counteract passive and active attacks," Workshop on Fault Diagnosis and Tolerance in Cryptography (FDTC), pp.3-17, 2008.

[7] D. El-Baze, J.B. Rigaud, and P. Maurine, "A Fully-Digital EM Pulse Detector," Design, Automation \& Test in Europe Conference \& Exhibition (DATE), pp.439-444, 2016.

[8] D. El-Baze, J.B. Rigaud, and P. Maurine, "An Embedded Digital Sensor Against EM and BB Fault Injection," Workshop on Fault Diagnosis and Tolerance in Cryptography (FDTC), pp.78-86, 2016.

[9] K. M. Zick, M. Srivastav, W. Zhang, and M. French, "Sensing nanosecond-scale voltage attacks and natural transients in fpgas," in Proceedings of the ACM/SIGDA international symposium on Field programmable gate arrays. , pp. 101-104, 2013.

[10] L. Zussa, J.M. Dutertre, J. Clediere, and B. Robisson,"Analysis of the fault injection mechanism related to negative and positive power supply glitches using an on-chip voltmeter", IEEE International Symposium on Hardware-Oriented Security and Trust (HOST), pp.130-135, 2014.

[11] M. Eisele, J. Berthold, D. Schmitt-Landsiedel, and R.Mahnkopf, "The Impact of Intra-Die Device Parameter Variations on Path Delays and on the Design for Yield of Low Voltage Digital Circuits," International Symposium on Low Power Electronics and Design, Digest, of Technical Papers, pp.237-242, 1996 